BIO Web of Conferences 2, 03006 (2014)

DOI: $10.1051 /$ bioconf/ 20140203006

(C) Owned by the authors, published by EDP Sciences, 2014

\title{
Complex molecules in the Orion Kleinmann-Low nebula
}

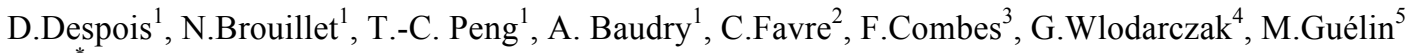 \\ et al. ${ }^{*}$ * \\ ${ }^{1}$ CNRS \& Univ. Bordeaux, LAB, UMR 5804, F-33270, Floirac, France \\ ${ }^{2}$ Dept. of Astronomy, Univ. Michigan, 500 Church St., Ann Arbor, MI 48109, USA \\ ${ }^{3}$ Observatoire de Paris,LERMA,CNRS, 61 Av. De l'Observatoire, 75014 Paris, France \\ ${ }^{4}$ PHLAM, Univ. Lille1, UMR 8523, 59655 Villeneuve d'Ascq Cedex, France \\ ${ }^{5}$ IRAM,Domaine Universitaire,300 rue de la Piscine, 38400 St Martin d'Hères, France
}

\begin{abstract}
In the framework of the delivery to the early Earth of extraterrestrial molecules, we have studied complex molecular species toward the Orion KleinmannLow nebula. This nebula is rich in molecules as well as in nascent stars and planetary systems. We focus here on $\mathrm{HCOOCH}_{3}, \mathrm{CH}_{3} \mathrm{OCH}_{3}$ and deuterated methanol. Upper limits on species of prebiotic interest like glycine were also obtained.
\end{abstract}

\section{Introduction}

Molecules important for the origin of life on Earth may have been brought to the early Earth in sizeable amounts by solar system small bodies (comets and asteroids) and their fragments (meteorites and micrometeorites). The molecular content of these small bodies is reminiscent of that of interstellar clouds from which stars and planetary systems form (e.g. Bockelée-Morvan et al 2000, A\&A 353,1101$)$. Note that to precise the role, possibly crucial, played in prebiotic chemistry by the extraterrestrial delivery of molecules, many difficult questions will have to be answered. For example, we need to understand the full chemical evolution from the original interstellar cloud to the evolved planetary disk and to determine quantitatively if extraterrestrial molecules reached the early Earth in sufficient amount.

We address here the question of the molecular complexity reached by interstellar chemistry, and of the formation processes of complex species. We selected for that purpose the Kleinmann-Low (KL) infrared nebula, located at the heart of the Orion Nebula. It is one of the richest regions in detected interstellar molecules and the host to many forming and young stars. The study of the spatial distribution of the molecules allows us to constrain their origin and their formation mechanism. Are the molecules produced in the gas-phase, on icy grain mantles, or through a combination of both processes ? Did shocks from the recent $(<1000 \mathrm{yr})$ explosive event play a role in their formation?

\footnotetext{
* In the context of the French Interdisciplinary Program EPOV we focus in this author list on scientists from French laboratories. We gratefully acknowledge the collaboration with our colleagues from Spain, Germany and USA: J. Cernicharo, B. Parise, A. Remijan, T. Wilson A. Wootten.
} 
We present selected results from a spectroscopic study of Orion KL with high spatial resolution (12",400-800 AU). The observations have been undertaken at millimetre wavelengths with the IRAM Plateau de Bure Interferometer and conducted for several years. We have studied methyl formate $\mathrm{HCOOCH} 3$ (ref. [1]), deuterated methanol $\left(\mathrm{CH}_{3} \mathrm{OD}\right.$ et $\mathrm{CH}_{2} \mathrm{DOH}$; ref. [2]), dimethyl ether $\mathrm{CH}_{3} \mathrm{OCH}_{3}$ (ref. [3,4]) and acetone $\mathrm{CH}_{3} \mathrm{COCH}_{3}$ (ref. [5]).

\section{Results}

Methyl formate $\mathrm{HCOOCH}_{3}$ (ref [1]). In addition to the determination of the physical properties of the gas (temperature, structure in space and velocity) this study has shown a likely association of the spatial distribution of this molecule with that of excited $\mathrm{H}_{2} 2.12 \mu \mathrm{m}$ emission, a tracer of the fast shock waves $(40-200 \mathrm{~km} / \mathrm{s})$ present in this region.

Deuterated methanol $\mathrm{CH}_{3} \mathrm{OD}$ and $\mathrm{CH}_{2} \mathrm{DOH}$ (ref.[2]). We have shown that the ratio $\mathrm{CH}_{2} \mathrm{DOH} / \mathrm{CH}_{3} \mathrm{OD}$ is rather constant over the region - this contrasts with the present day spread in physical conditions and suggests a formation and storage of deuterated methanol (and, likely, of methanol itself) in the ice mantles of the interstellar grains at a time where the nebula was more homogeneous. The value of the ratio we found, $<1$, confirms the strong difference between Orion KL and the surroundings of low-mass stars found by Rataczack et al (2011, A\&A 528, L13).

Dimethyl ether $\mathrm{CH}_{3} \mathrm{OCH}_{3}$ (ref. [3,4]). Comparaison of $\mathrm{CH}_{3} \mathrm{OCH}_{3}$ with $\mathrm{HCOOCH}_{3}$ has shown a remarkable correlation of their spatial distributions - which is not found with ethanol $\mathrm{CH}_{3} \mathrm{CH}_{2} \mathrm{OH}$ or with formic acid $\mathrm{HCOOH}$ (the latter is anticorrelated). The simplest interpretation is that both species are formed from a same precursor. Two main models have been proposed to form these species : on grain mantles (e.g. Bisschop et al 2007, A\&A 465,913; Oberg et al 2010, ApJ 716, 825) or in the gas phase (e.g. Neill et al 2010, J.Ph.Ch. A 115, 6472). The precursor would be respectively $\mathrm{CHO} \bullet$ or $\mathrm{CH}_{3} \mathrm{OH}_{2}^{+}$.

In the latter case $\mathrm{HCOOCH}_{3}$ is formed from $\mathrm{HCOOH}$. Another reaction producing $\mathrm{HCOOCH}_{3}$ from $\mathrm{H}_{2} \mathrm{CO}$ has been proposed (Blake et al 1988, Lect. Note. Phys 315,132 ), but has been shown to have a too high energy barrier $(128 \mathrm{~kJ} / \mathrm{mol} \sim 15000 \mathrm{~K} \sim 1.2 \mathrm{eV})$. Could the kinetic energy provided in a shock $\left(\mathrm{V}_{\text {shock }} \sim 30-40 \mathrm{~km} / \mathrm{s}\right)$ overcome the energy barrier and make this reaction possible?

"Prebiotic" species. Some molecules of direct interest for prebiotic chemistry, a pre-sugar $\mathrm{CH}_{2} \mathrm{OHCHO}$ (glycolaldehyde), aminoacetonitrile $\mathrm{NH}_{2} \mathrm{CH}_{2} \mathrm{CN}$ (a precursor of glycine) and the simplest amino acid, glycine itself $\mathrm{NH}_{2} \mathrm{CH}_{2} \mathrm{COOH}$, have been searched for, but only upper limits have been obtained (Guélin et al. 2008, Ap\&SS 313,45; ref [1]). Note that the first two species have been found in other young stellar environments.

\section{References}

1. C. Favre, D. Despois, N. Brouillet, A. Baudry, F. Combes, M. Guélin, A. Wootten and G. Wlodarczak, A\&A 532, A32 (2011)

2. T.-C. Peng, D. Despois, N. Brouillet, B. Parise, and A. Baudry, A\&A 543, A152 (2012)

3. C. Favre, H. A. Wootten, A. J. Remijan, N. Brouillet, T. L. Wilson, D. Despois, and A. Baudry, ApJ.Lett. 739, L12 (2011)

4. N. Brouillet, D. Despois, A. Baudry, T. -C. Peng, C. Favre, A. Wootten, A. J. Remijan, T. L. Wilson, F. Combes, and G. Wlodarczak, A\&A 550, A46 (2013)

5. T.-C. Peng, D. Despois, N. Brouillet, A. Baudry, C. Favre, A. Remijan, A. Wootten, T. L. Wilson, F. Combes, and G. Wlodarczak, A\&A (subm.) 